\title{
Security authentication using phase-encoded nanoparticle structures and polarized light
}

\author{
Artur Carnicer, ${ }^{1,2, *}$ Amir Hassanfiroozi, ${ }^{3}$ Pedro Latorre-Carmona, ${ }^{1,4}$ Yi-Pai Huang, ${ }^{3}$ and Bahram Javidi ${ }^{1}$ \\ ${ }^{1}$ Electrical and Computer Engineering Department, University of Connecticut, 371 Fairfield Road, Storrs, Connecticut 06269, USA \\ ${ }^{2}$ Universitat de Barcelona (UB), Facultat de Física, Departament de Física Aplicada i Òptica, Martí i Franquès 1, 08028 Barcelona, Spain \\ ${ }^{3}$ Department of Photonics, Display Institute, National Chiao Tung University, 1001 Ta Hsueh Rd., CPT Bldg., 300 Hsinchu, Taiwan \\ ${ }^{4}$ Institute of New Imaging Technologies, Universitat Jaume I, Campus Riu Sec s/n, 12071 Castelló de la Plana, Spain \\ ${ }^{*}$ Corresponding author: artur.carnicer@ub.edu
}

Received October 16, 2014; revised November 24, 2014; accepted November 25, 2014; posted November 26, 2014 (Doc. ID 225126); published January 7, 2015

\begin{abstract}
Phase-encoded nanostructures such as quick response (QR) codes made of metallic nanoparticles are suggested to be used in security and authentication applications. We present a polarimetric optical method able to authenticate random phase-encoded QR codes. The system is illuminated using polarized light, and the QR code is encoded using a phase-only random mask. Using classification algorithms, it is possible to validate the QR code from the examination of the polarimetric signature of the speckle pattern. We used Kolmogorov-Smirnov statistical test and Support Vector Machine algorithms to authenticate the phase-encoded QR codes using polarimetric signatures. (C) 2015 Optical Society of America

OCIS codes: (100.4998) Pattern recognition, optical security and encryption; (260.5430) Polarization; (100.0100) Image processing.

http://dx.doi.org/10.1364/OL.40.000135
\end{abstract}

Optics and photonics technologies have been investigated for security and encryption [1-12]. Optical methods provide large bandwidth, many degrees of freedom, small wavelengths, and multidimensional keys, among other benefits for security applications. Many approaches have been proposed for using information optics for authentication, security, verification, and encryption [3-7].

Quick response (QR) codes are a ubiquitous technology that is being used for a variety of applications. QR codes are vulnerable to the possibility of including harmful information. To remedy this problem, it has been suggested to print the code using some special nanoparticle inks [13] or to use random phase-only tags to validate a QR code optically [14].

In this report, we propose the use of $\mathrm{QR}$ codes made of metal nanoparticles for security and authentication applications. Our target is to develop an optical system able to authenticate QR codes without accessing the included information. To improve security, the QR code is encoded using a phase-only random mask so that the propagated signal becomes a speckle-noise distribution. The sample is illuminated with a polarized beam, and then the scattered light is analyzed. Using classification algorithms such as multiclass support vector machine (SVM) it is possible to validate a $\mathrm{QR}$ code from the examination of the speckle pattern.

Figure 1 shows the three different samples used in the experiments. Figures 1(a) and 1(b) display two $21 \times 21$ pixels $\mathrm{QR}$ codes. The size of these codes is $0.840 \times 0.840 \mathrm{~mm}^{2}$, with the pixel size of $40 \mu \mathrm{m}$. The sample displayed in Fig. 1(a) is made of gold nanoparticles (size 30-50 nm) deposited on a glass surface. The code of Fig. 1(b) is made using a different technique. First, a film of platinum is sputtered on the glass substrate, and then a silicon nitride layer is deposited on the first one. This second coating is etched according to a predetermined pattern so the information is coded. Notice that it was intended that both $\mathrm{QR}$ codes encoded the same information. The encoded message is the sequence 12 15522 13. On the other hand, the sample of Fig. 1(c) was generated using gold nanoparticles, but no QR code is implemented.

The samples are analyzed using linearly polarized light by means of the optical setup depicted in Fig. 2. A green laser diode $(\lambda=532 \mathrm{~nm})$ is used as a light source. The intensity of the field is controlled by means of a variable neutral density filter. At this point, the beam is circularly polarized in order to illuminate the sample with the same amount of energy irrespective of the direction of polarization of the incident light. Then, the beam is linearly polarized and passes through the QR code. The scattered light is propagated and subsequently recorded on an 8-bit CCD camera placed $15 \mathrm{~mm}$ away from the sample. In order to enhance security, a random phase-only key code mask can be attached to the sample. The polarizer is rotated from $0^{\circ}$ to $179^{\circ}$ at intervals of one degree, and one image is taken for each polarization direction $(P)$. Figure $\underline{3}$ shows the propagated light distributions for the three samples $\left(P=0^{\circ}\right)$ at the camera plane.

Figure 4 shows the histograms of the propagated images for several directions of polarization of the illumination beam. Note that the variable neutral density filter is set in a way that the camera never saturates for the complete set of measures. It is noticeable that for each sample, the histograms are slightly different depending on the polarization of the incident light.
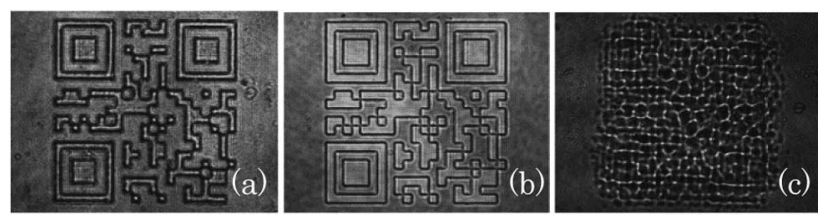

Fig. 1. (a) Gold nanoparticles QR code (sample A), (b) platinum-based QR code (sample B), and (c) gold nanoparticles structure without QR mask (sample C). 


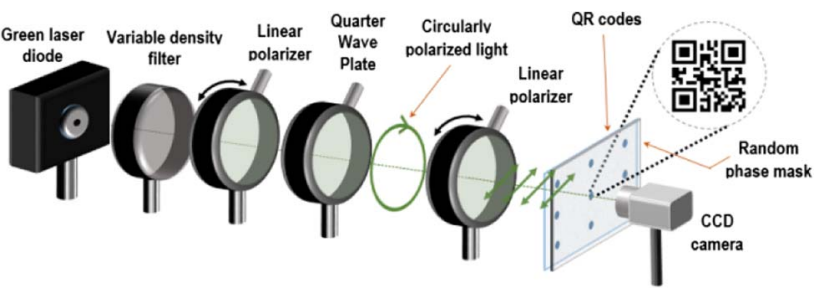

Fig. 2. Optical setup.
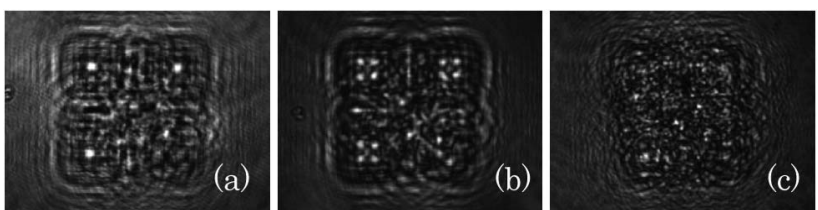

Fig. 3. Propagated QR codes: left, sample A; center, sample B; and right, sample $\mathrm{C}$ (see Fig. $\underline{1}$ for details).
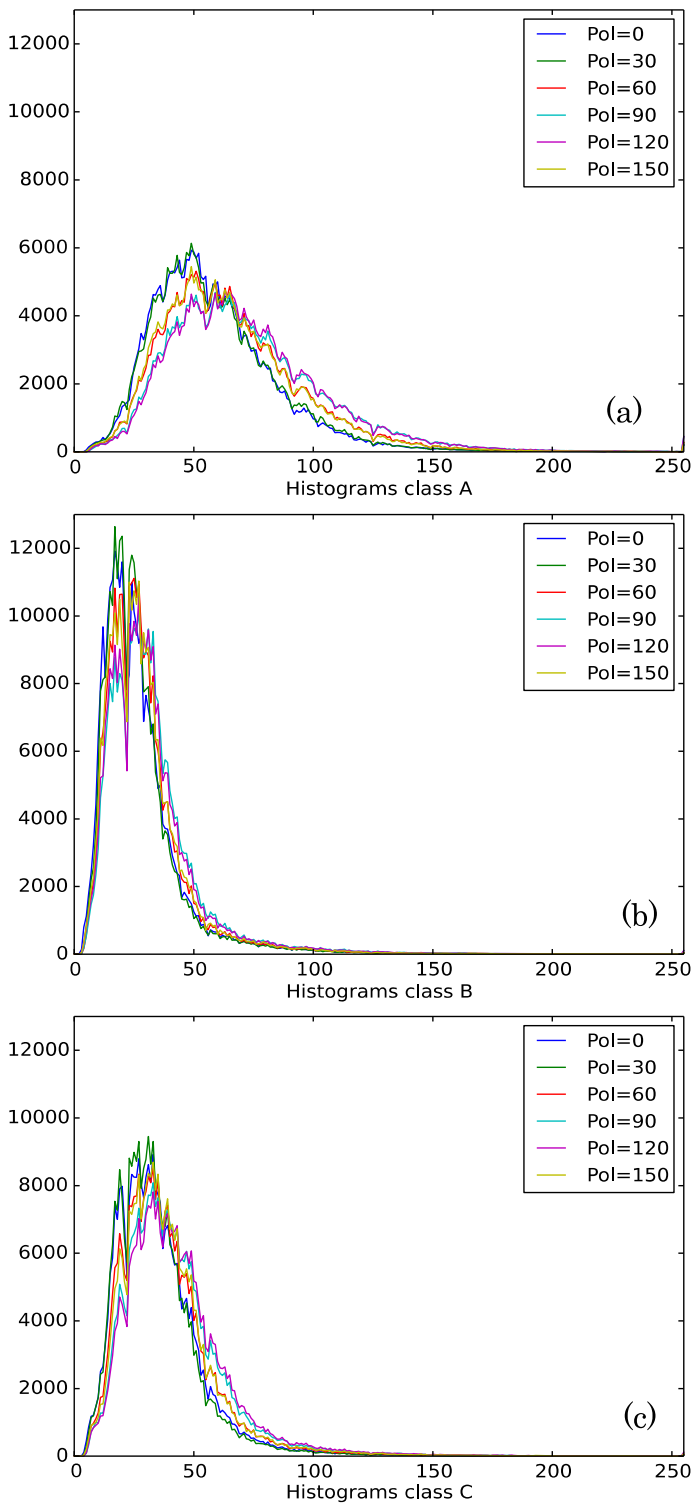

Fig. 4. Histograms of the three samples in Figs. 1(a)-1(c) for different directions of polarization $\left(0^{\circ}, 30^{\circ}, 60^{\circ}, 120^{\circ}\right.$, and $\left.150^{\circ}\right)$.
This performance is related with the dichroic behavior of metallic nanoparticles. In other words, the absorption coefficient depends on the polarization direction of the incident light, and thus the recorded light distributions and their corresponding histograms change accordingly. Some studies on nanoparticles dichroism can be found in Refs. [15-17].

The information obtained from the 180 histograms for each QR code is used for classification purposes. The histogram is considered as the input feature vector for each sample in order to determine the corresponding class. Due to the high dimensionality of the feature vectors, principal component analysis (PCA) is used in order to reduce it [18]. Figure 5 shows a 3D plot with the first three eigenvectors (those that explain the highest amount of variance in the data) of the PCA decomposition. This plot shows that the three classes are clearly separable. Nevertheless, the information is not secure, so anyone can access it. For instance, light distributions from Fig. 2 could be back-propagated using the Fresnel diffraction integral. From the resulting distribution, it would be possible to infer some information in the QR code.

Phase-encoding the $\mathrm{QR}$ code using double or single random-phase encoding is a possible way to circumvent this problem $[\underline{4}, 5]$. This is equivalent to multiplying the QR code by a phase-only random mask. After propagation, the wave-front becomes a noise-like distribution, thus the encoded signal becomes indistinguishable [14]. Phase-encoded signals can be easily produced by attaching a diffusor such as adhesive tape to the $\mathrm{QR}$ code. Figure 6 shows the propagated phase-encoded QR codes. The recorded signals are nearly identical, and therefore the classification problem becomes more complex.

Figure 7 shows some recorded histograms for the three phase-encoded samples using an adhesive diffuser tape. These curves look different when compared

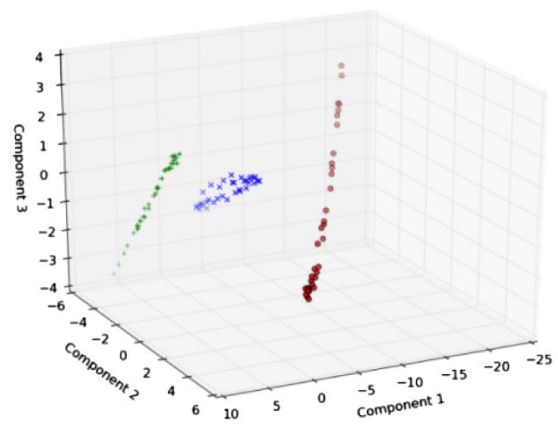

Fig. 5. PCA: red, sample A; green, sample B; and blue, sample C.
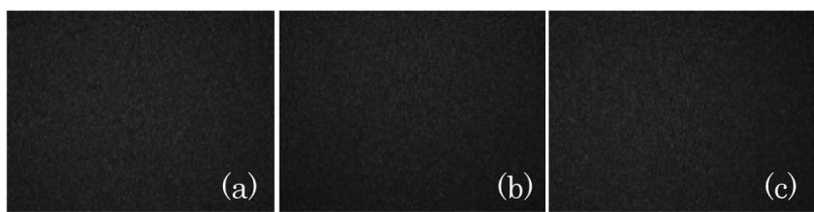

Fig. 6. Propagated random phase-encoded QR codes: (a) sample A, (b) sample B, and (c) sample C. 

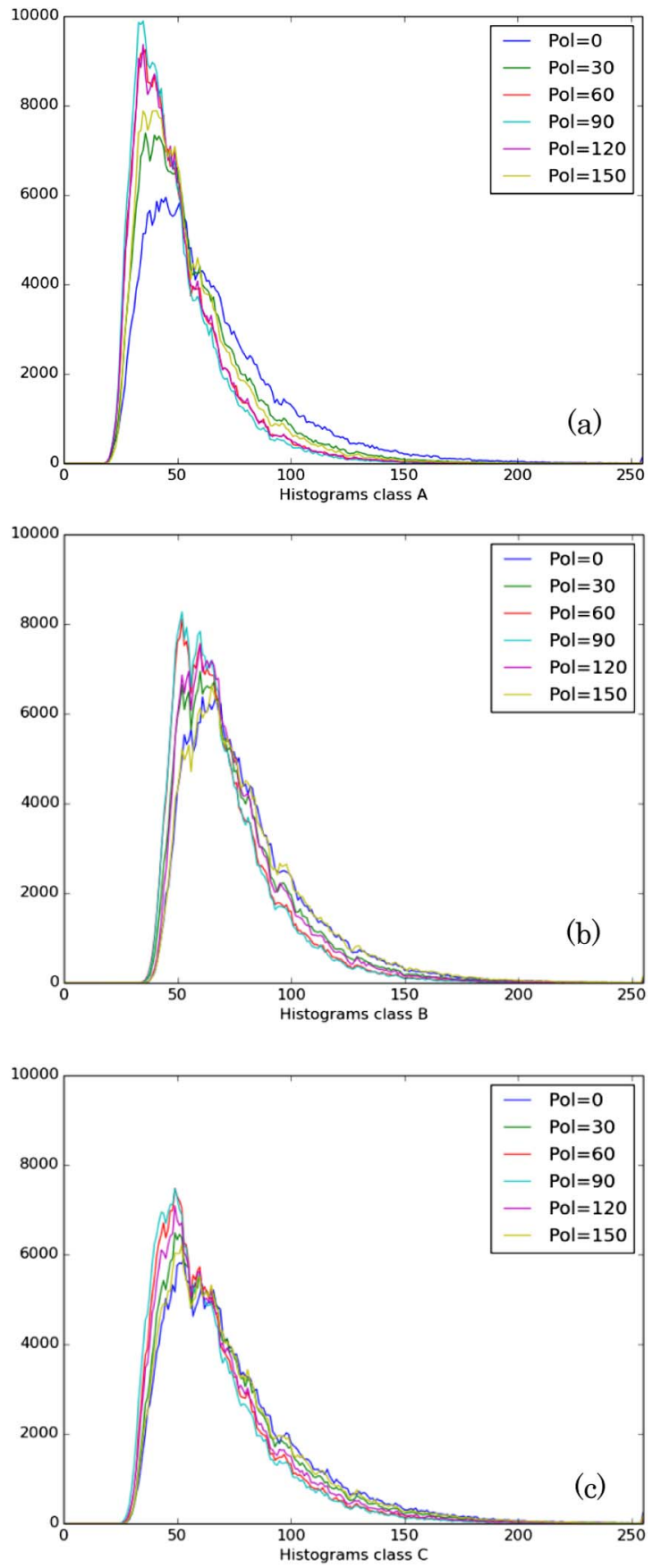

Fig. 7. Histograms of the three phase-encoded samples in Figs. 1(a) -1 (c) for different directions of polarization $\left(0^{\circ}\right.$,

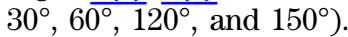

with the corresponding histograms obtained for the nonphase-encoded samples (see Fig. 4).

On the one hand, the profile of each histogram depends on the polarization direction of the incident beam. These profiles are similar for the three samples considered. On the other hand, the diffusor generates a speckle-like pattern, and consequently the histograms are expected to exhibit a Gamma-like distribution profile

$$
\Gamma(I)=\left(\frac{n_{0}}{\bar{I}}\right)^{n_{0}} \frac{I^{n_{0}-1} \exp \left(-I n_{0} / \bar{I}\right)}{\Gamma\left(n_{0}\right)} \quad n_{0}=\left(\frac{\bar{I}}{\sigma}\right)^{2},
$$

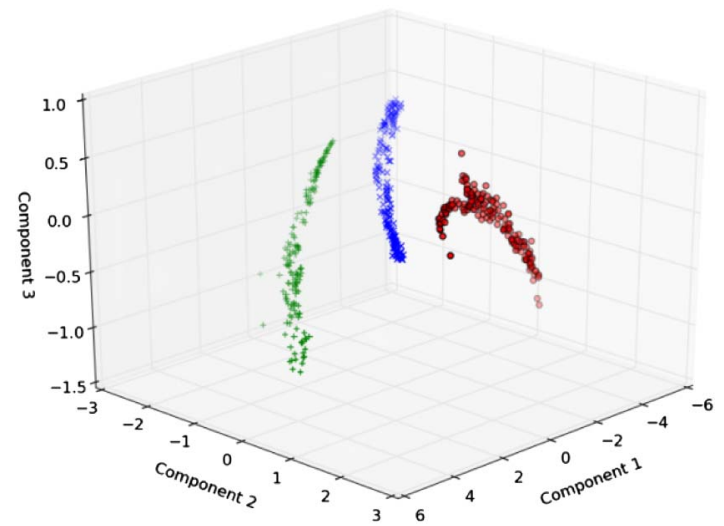

Fig. 8. Principal components analysis for the phase encoded samples. Red, sample A; green, sample B; and blue, sample C.

where $I, \bar{I}$, and $\sigma$ are the intensity data points, its average and the corresponding standard deviation, respectively [19]. We performed a Kolmogorov-Smirnov test to measure the distance between the histograms and the Gamma distribution [20]. In all the cases considered, this test indicates that the obtained histograms are compatible with a speckle Gamma distribution. Finally, it is worth to point out that despite the obvious similarities among the histograms displayed in Figs. 4(a) $-4(\mathrm{c})$, the histograms present some subtle differences that are sufficient for distinguishing among the three samples.

As in the previous case, PCA was applied on the sample histograms. A representation using the first three eigenvectors shows again that the three classes are separable (Fig. 8).

We used a multi-class SVM approach for the classification of the different types of samples into three classes using the whole 256-dimensional features corresponding to the sample histograms. SVM has attracted much attention in the last years because of its competitive classification results in high dimensional low cardinality (i.e., number of samples) classification problems. In particular, we used the approach implemented in the LIBSVM package [21].

A short explanation about how SVM works is as follows. Input data are transformed to have zero mean and standard deviation equal to one, before running SVM. This transform allows the classification to be insensitive to data scale. Multiclass SVM generates $N_{c}$ $\left(N_{c}-1\right) / 2$ two-class SVM classifiers, where $N_{c}$ is the total number of classes. Each one is trained considering data from two of the classes.

Let us consider $t$ training data given by $\left\{\left(x_{m}, y_{m}^{i j}\right)\right\}_{m=1 \ldots . . t}$, where $x_{m} \in \mathrm{R}^{n}$ is the histogram, and $y_{m}^{i j} \cdot \in\{-1,+1\}$ represents an arbitrary label assigned to the two classes, $i$ and $j$. For the "one-against-one" problem, the following classification problem is solved:

$$
\min _{\omega^{i j}, b^{i j}, \xi^{i j}}\left\{\frac{1}{2}\left\|\omega^{i j}\right\|^{2}+C \sum_{m=1}^{t} \xi_{m}^{i j}\right\}
$$

restricted to

$y_{m}^{i j} \cdot\left\langle\phi\left(x_{m}\right), \omega^{i j}\right\rangle+b^{i j} \geq 1-\xi_{m}^{i j}, \quad \forall m=1, \ldots, t$

$\xi_{m}^{i j} \geq 0$, 
Table 1. SVM Results, Three-Class Problem

\begin{tabular}{lcc}
\hline Phase Mask & Accuracy (Test Set, in \%) & No. of Test Runs \\
\hline No & 100 & 40 \\
Yes & 100 & 40 \\
\hline
\end{tabular}

where $\omega^{i j}$ and $b^{i j}$ define a maximum margin linear classifier in the feature space for each two-class problem; $\xi_{t}^{i j}$ are positive slack variables to deal for permitted errors in the classification; and the symbols $\langle$,$\rangle and \|\|$ stand for the dot product and the Euclidean norm, respectively. Function $\phi$ is a nonlinear mapping to a higher dimensional space, and $C$ is the penalty parameter. It can be shown that solving Eq. (2) with the restrictions imposed by Eq. (3) generates the following decision function to be used for any sample $x^{\prime}$ that belongs to the test set [22]:

$$
f\left(x^{\prime}\right)=\operatorname{sign}\left(\sum_{m=1}^{t} \alpha_{m}^{i j} \cdot y_{m}^{i j} \cdot K\left(x_{m}, x^{\prime}\right)+b^{i j}\right) .
$$

$\alpha_{m}^{i j}$ are Lagrange multipliers found by optimization, and $K\left(x_{m}, x^{\prime}\right)=\left\langle\phi\left(x_{m}\right), \phi\left(x^{\prime}\right)\right\rangle$ is the so-called kernel function. In our case, a radial basis function kernel type was used, $K\left(x_{m}, x^{\prime}\right)=\exp \left(-\gamma\left\|x_{m}-x^{\prime}\right\|^{2}\right)$, where $\gamma$ is a scaling parameter. Notice that the sign in Eq. (4) defines the class the sample $x^{\prime}$ belongs to.

SVM is generalized to $N_{c}$ classes, by defining $N_{c}\left(N_{c}-\right.$ 1) $/ 2$ decision functions. Then, a voting strategy is considered to establish the class to which each histogram belongs. In particular, a vote is given to the class the test sample is assigned for each of the $N_{c}\left(N_{c}-1\right) / 2$ two-class classification problems. The final class of the sample is the one with the largest number of votes. For further details, the reader is referred to [23].

The data set was divided into training and test sets, using a hold-out strategy. The best combination of parameters $(C, \gamma)$ must be found on the training set. They were obtained using a $k$-fold cross-validation strategy $(k=5)$ over the training set. The figure of merit used for the assessment of the classification quality (over the test set) is the Overall Accuracy measure, defined as the total number of correctly classified samples divided by the total number of test samples [24].

Several tests were performed to check the classification capabilities of SVM. First, a three-class classification problem is considered (see Table 1). When QR codes are not phase encoded, SVM provides error-free results. For the phase-encoded QR codes, the accuracy for the classification method is also $100 \%$. Due to the random selection of the training and test sets, the test was run 40 times. For completeness, two class problems were also considered (see Table 2). Training the system using histograms from samples $\bar{A}$ and B provides $100 \%$ accuracy. The same happens when codes $\mathrm{B}$ and $\mathrm{C}$ or $\mathrm{A}$ and $\mathrm{C}$ are taken into account. If the $\mathrm{QR}$ codes are phase encoded, classification is always error free for the two class classification problem.
Table 2. SVM Results, Two-Class Problem ${ }^{a}$

\begin{tabular}{lcc}
\hline QR Codes & Accuracy (Test Set, in \%) & No. of Test Runs \\
\hline A and B & 100 & 40 \\
B and C & 100 & 40 \\
A and C & 100 & 40 \\
\hline
\end{tabular}

${ }^{a}$ Phase encoded QR masks.

In summary, we have proposed the use of polarimetric measurements for authentication of phase encoded QR codes made of metal nanoparticles for security applications. Using polarized light and multi-class SVM, nanoparticle QR codes can be correctly classified. The codes can be encoded using a variety of random-phase distributions for security and encryption. The classification algorithms are able to distinguish among different samples from the speckle patterns analysis.

\section{References}

1. O. Matoba, T. Nomura, E. Perez-Cabre, M. S. Millan, and B. Javidi, Proc. IEEE 97, 1128 (2009).

2. A. Alfalou and C. Brosseau, Adv. Opt. Photon. 1, 589 (2009).

3. W. Chen, B. Javidi, and X. Chen, Adv. Opt. Photon. 6, 120 (2014).

4. P. Réfrégier and B. Javidi, Opt. Lett. 20, 767 (1995).

5. O. Matoba and B. Javidi, Opt. Lett. 24, 762 (1999).

6. Y. Li, K. Kreske, and J. Rosen, Appl. Opt. 39, 5295 (2000).

7. B. Javidi and T. Nomura, Opt. Eng. 39, 2031 (2000).

8. X. Tan, O. Matoba, Y. Okada-Shudo, M. Ide, T. Shimura, and K. Kuroda, Appl. Opt. 40, 2310 (2001).

9. P. Clemente, V. Durán, V. Torres-Company, E. Tajahuerce, and J. Lancis, Opt. Lett. 35, 2391 (2010).

10. E. Pérez-Cabré, M. Cho, and B. Javidi, Opt. Lett. 36, 22 (2011).

11. T. Yatagai, T. Ochiai, and D. Barada, J. Opt. 14, 094005 (2012).

12. J. F. Barrera, A. Mira, and R. Torroba, Opt. Express 21, 5373 (2013).

13. J. M. Meruga, W. M. Cross, P. S. May, Q. Luu, G. A. Crawford, and J. J. Kellar, Nanotechnology 23, 395201 (2012).

14. A. Markman, B. Javidi, and M. Tehranipoor, IEEE Photon. J. 6, 1 (2014).

15. W. Gotschy, K. Vonmetz, A. Leitner, and F. R. Aussenegg, Opt. Lett. 21, 1099 (1996).

16. B. Canfield, S. Kujala, K. Jefimovs, J. Turunen, and M. Kauranen, Opt. Express 12, 5418 (2004).

17. U. Guler and R. Turan, Opt. Express 18, 17322 (2010).

18. W. R. Dillon and M. Goldstein, Multivariate Analysis: Methods and Applications (Wiley, 1984).

19. J. C. Dainty, Prog. Optics 14, 1 (1977).

20. F. Massey, Jr., J. Am. Stat. Assoc. 46, 68 (1951).

21. C. C. Chang and C. J. Lin, ACM TIST 2, 27 (2011).

22. B. Schölkopf and A. Smola, Learning With Kernels-Support Vector Machines, Regularization, Optimization and Beyond (MIT, 2002).

23. C. W. Hsu and C. J. Lin, IEEE Trans. Neural Netw. 13, 732 (2002).

24. N. Japkowicz and M. Shah, Evaluating Learning Algorithms (Cambridge University, 2011). 